\title{
Intégration/ségrégation : une clé de lecture des recompositions des modèles de développement ? Le cas de la Nouvelle-Calédonie
}

\author{
Séverine Bouard ${ }^{1}$, Jean-Michel Sourisseau ${ }^{2}$, Benoit Zenou ${ }^{3}$ \\ 1 Géographe, IAC (Institut agronomique néo-calédonien), Nouvelles ruralités et destin commun, 98825 Pouembout, \\ Nouvelle-Calédonie \\ 2 Socioéconomiste, CIRAD, UMR ART-Dev, 34398 Montpellier cedex 5, France \\ 3 Socioéconomiste, CIRAD, Direction scientifique, 34398 Montpellier cedex 5, France
}

Éviter les dualismes - que reproduisent sans doute inévitablement les institutions - est une vertu de la pensée. Cet article, consacré au développement agricole de la Nouvelle-Calédonie, tire son intérêt de l'analyse des pratiques qui se développent à l'ombre de la dualité intégration/ségrégation. Si les politiques publiques aboutissent à promouvoir une sorte de développement séparé pour certaines communautés, les acteurs concernés peuvent s'en saisir pour mieux s'intégrer aux dynamiques dominantes auxquelles un traitement uniforme de leur situation ne leur aurait pas donné accès. Mais il montre aussi que, dans ce mouvement, ce sont des configurations originales, spécifiques, voire innovantes, de relations au marché et aux institutions qui s'esquissent. Cet article éclaire ainsi la subtilité des stratégies qui redéfinissent les particularités dans une globalisation marchande. Il donne ainsi à réfléchir aux relations entre local et global qui sont au cœur de la question du développement durable.

La Rédaction

Mots-clés :

intégration ;

ségrégation ;

politiques de

développement ;

Nouvelle-Calédonie

Keywords:

integration;

segregation;

development policies;

New Caledonia
Résumé - Cet article propose une clé de lecture originale articulant les notions d'intégration et de ségrégation, pour approfondir les débats sur les modèles de développement notamment en milieu rural. Les controverses opposent souvent un modèle de développement fondé sur une agriculture spécialisée et productiviste, connectée aux marchés globalisés et un modèle de développement local associé à des filières de proximité et à des formes de production agricole favorisant l'intensification écologique et la cohésion sociale. Dans un premier temps, l'intérêt heuristique des notions d'intégration et de ségrégation pour lire les recompositions des modèles de développement est précisé. La clé de lecture est ensuite testée dans le cas de la Nouvelle-Calédonie, emblématique d'une situation coloniale de peuplement dans laquelle l'action publique se réfère à des modèles duaux de développement. Nous insistons sur le caractère stratégique des segmentations des politiques et des territoires associés, et montrons que les frontières entre ces territoires sont le lieu d'innovation où se construisent les modèles en devenir.

\begin{abstract}
Integration/segregation: theoretical tools for a better understanding of evolution in development models? The case of New Caledonia. This paper proposes an original theoretical framework in order to deepen the debate about development models, especially in rural areas. Dichotomous views dominate the debate: a specialized and productivist agricultural model linked to globalized markets vs a local development model associated with local supply chains or quality niches and to types of agricultural production conducive to ecological intensification and social cohesion. This paper identifies how concepts of integration and segregation provide original theoretical tools in order to analyze these debates in depth. The theoretical framework is tested in the case of New Caledonia, through the analysis of rural development policies. This emblematic example of a colonial settlement is interesting. It offers public policies based on segregated models of development, but which simultaneously pursue a global objective to overcome the historical segregation between communities, land-use, etc. The field study allows the authors to highlight the strategic dimension of the segmentation
\end{abstract}

Auteur correspondant : S. Bouard, bouard@iac.nc 
of policies and of the targeted territories. At the local scale, the study of Kanak development strategies shows that the boundaries between these different kinds of development territories are places where new development models and innovations can arise. Despite some limitations, the theoretical framework is particularly suited to understanding the historical and current transformations of the archipelago and offers original prospects for generalization. Mainly, the heuristic strength of the framework is not based on the opposition, but on the identification of connections between integration and segregation.

Dans tout processus de développement rural, les changements affectant les relations entre agricultures, ruralités et sociétés sont générateurs d'exclusions, d'instabilité et de conflits politiques et sociaux. Les objectifs du Millénaire (United Nations, 2000) témoignent de la prise de conscience internationale de ces enjeux, ainsi que de l'urgence d'y répondre. Mais la compréhension des transformations des Suds et les solutions visant le développement de leurs sociétés font souvent l'objet de visions dichotomiques. Ainsi les oppositions entre «petites » et «grandes » exploitations ou entre agricultures familiales et entrepreneuriales animent les débats sur la compétitivité des structures de production (Hazell et al., 2007 ; IAASTD, 2009 ; Timmer, 2009) ; la nature inclusive ou exclusive des modèles agricoles est au cœur des représentations portées par la Banque mondiale sur les dynamiques rurales (World Bank, 2007 ; GibsonGraham, 2010 ; Losch et al., 2011,).

Récemment, deux représentations du développement susceptibles d'incarner et de mettre en débat ces dualités ont émergé : l'intégration et la ségrégation (I/S) (Hubert et Caron, 2009 ; Hubert, 2010). Parfois explicitement formulées, mais souvent présentes en creux dans les modèles proposés pour décrire les recompositions des mondes ruraux, elles opposent en cela bien souvent les chercheurs (Zenou, 2011). Pour certains, le développement passe par la spécialisation des espaces et formes de production pour intensifier et par le traitement des effets négatifs de cette "ségrégation", quel que soit le coût des compensations. Pour d'autres, «l'intégration" des fonctions multiples assignées à l'agriculture, mais aussi des communautés présentes sur un même territoire, est globalement préférable. Au-delà des avantages économiques qu'elle procure, elle maintient un tissu social, contribue à la gestion raisonnée des ressources territoriales.

Cet article examine en quoi ces notions aident à décrire et comprendre les dynamiques spatiales, sociétales et culturelles des territoires ruraux et leur mise en politique. Pour cela, après avoir défini ce que nous entendons par intégration/ségrégation (I/S) et la nature de leur relation dialectique, nous illustrons la pertinence de cette clé de lecture pour l'analyse des politiques de développement rural de la Nouvelle-Calédonie. Malgré quelques limites, le couple I/S se révèle particulièrement adapté pour comprendre les transformations historiques et actuelles de l'archipel et offre d'intéressantes perspectives de généralisation.

\section{Le couple intégration/ségrégation comme clé de lecture : vers une analyse plus systémique du développement}

Les notions d'intégration et de ségrégation sont depuis longtemps utilisées pour décrire et comprendre des phénomènes observables touchant au développement, notamment en ce qui concerne les Suds. Elles sont par exemple utilisées pour l'analyse des dynamiques urbaines (Cary et Fol, 2012), des articulations entre rural et urbain (Hubert, 2010), des mobilités économiques (Graner, 2008 ; Léonard, 2005), de la cohabitation entre élevage et agriculture (Gautier et al., 2005), des inégalités économiques et démographiques des territoires (Scholz, 2005 ; Sencébé 2009), ou plus largement des modèles $\mathrm{du}$ développement agricole et de leurs frontières (Van der Ploeg, 2000 ; Caron et Le Cotty, 2006).

Issues du sens commun, les notions d'intégration et de ségrégation ont l'avantage de donner à voir des processus complexes, tandis que leur opposition, très parlante elle aussi, permet de décrire les sociétés et leur environnement. Cependant, il est nécessaire de prendre de la distance vis-à-vis de leur dimension normative, qui conduit souvent à concevoir l'intégration comme positive et la ségrégation comme négative, et d'aller plus loin dans l'explicitation de leurs soubassements sémantiques et théoriques.

D'un point de vue sémantique, les termes « intégration » et "ségrégation » renvoient, selon les objets d'étude, à des théories et des approches différentes. Les chercheurs en utilisent donc parfois d'autres qui renvoient à des états/dynamiques semblables, mais tentent d'éviter toute connotation : ainsi en est-il des termes connexes tels que l'inclusion (Zidouri, 2008) ou la déségrégation (Morin, 1966) pour l'intégration, et l'exclusion (Elias et Scotson, 1997 ; Moustier et Anh, 2010), la stratification (Hubert, 2010), la segmentation (Vieillard-Baron et De Almeida Vasconcelos, 2004) ou la fragmentation (Hobbs et al., 2008) pour la ségrégation. Ce dernier terme de «fragmentation » est en particulier devenu le nouveau paradigme des études du développement. Cette notion répondrait à la remise en question des grandes théories du développement (Menzel, 1992) ; celles renvoyant à l'évolutionnisme et principalement fondées sur la croissance, mais aussi le développement durable, jugé comme un simple prolongement des logiques et acceptations passées du développement (Bullard, 2011). Au sens de Scholz (2005), la fragmentation est entendue comme 
un principe de structuration causée par des processus de transformation politique et sociale en lien avec la mondialisation. Dans cette perspective, la fragmentation a remplacé la notion de polarisation et apparaît comme une nouvelle forme de ségrégation à plus grande échelle.

Au-delà de la sémantique, les acceptions de l'intégration (davantage que de la ségrégation) diffèrent selon les disciplines examinant les questions de développement. En économie, l'intégration est entendue comme la participation renforcée aux marchés, notamment internationaux - ce qui suppose une double intégration, nationale et globale. C'est du reste cette attention portée à la globalisation qui permet de distinguer, en économie du territoire, le développement local du développement territorial (Koop et al., 2010). En géographie sociale, l'intégration s'entend davantage au sens des territoires eux-mêmes et des sociétés les faisant vivre. C'est plutôt ainsi que nous l'abordons ici.

Malgré les divergences et prudences sémantiques, $\mathrm{du}$ fait de leurs utilisations nombreuses et des débats théoriques sous-jacents, il semble que les notions d'intégration et de ségrégation sont utiles pour décrire des processus en tenant compte de leur dimension systémique. Ainsi, si l'on adopte une vision processuelle élargie permettant de rendre possible une discussion pluridisciplinaire, nous pouvons définir l'intégration (et par opposition la ségrégation) comme un processus d'association (et par opposition de dissociation), volontaire ou involontaire, d'un espace, d'une ressource, d'un acteur ou d'une idée, avec un autre espace, une autre ressource, un autre acteur ou une autre idée.

Cependant, pour que le couple intégration/ségrégation devienne pertinent comme clé de lecture des dynamiques rurales, trois limites doivent être dépassées et nous proposons pour chacune d'elles une hypothèse de travail :

- Premièrement, les dynamiques apparaissant intégrationnistes peuvent devenir ségrégationnistes par changement des représentations sociétales (et réciproquement $^{1}$ ).

Hypothèse 1 : les deux fonctionnent ensemble et non de façon dichotomique. Il convient de les concevoir comme les faces d'un prisme permettant de révéler et de caractériser des phénomènes.

- Deuxièmement, intégration et ségrégation renvoient à des conceptions différentes selon que l'on se situe dans une approche statique ou dynamique des phénomènes. Si l'intégration ou la ségrégation peuvent être considérées comme des finalités, nous préférons les appréhender comme des moyens et/ou des processus permettant

\footnotetext{
1 Ainsi les labels comme Max Havelaar offrent la possibilité à des agriculteurs des Suds d'accéder au commerce mondial.
}

d'atteindre d'autres objectifs (bien-être, réduction de la pauvreté, etc.).

Hypothèse 2 : les logiques d'intégration et de ségrégation peuvent apparaître dans un processus d'action et de réaction ; chaque processus d'intégration produit partiellement de la ségrégation et réciproquement, ce qui permet d'en comprendre mieux les logiques complexes.

- Troisièmement, selon l'échelle d'analyse adoptée par le chercheur, un même phénomène peut être décrit comme intégré ou ségrégué.

Hypothèse 3 : les frontières définissant les espaces intégrés ou ségrégués sont généralement poreuses ; cette porosité se traduit par des recompositions et influence les processus d'intégration et de ségrégation. S'intéresser aux frontières permet d'éclairer les phénomènes d'hybridation.

Ces trois hypothèses sont testées dans le cadre de l'analyse du développement rural calédonien, en particulier kanak. En effet, la colonisation de peuplement a été marquée par le bagne, le développement agricole (dans un modèle pionnier d'accaparement foncier) et l'exploitation minière. Elle a notamment conduit au développement de plusieurs formes de ségrégation envers le monde autochtone kanak (Saussol, 1979; Merle, 1995). Leur reconnaissance dessine aujourd'hui un modèle original de décolonisation négociée. Les accords politiques de Matignon-Oudinot (1988) et de Nouméa (1998), tout en scellant la permanence d'une profonde dualité entre Calédoniens d'origine européenne et Kanak, génèrent de nouvelles formes de ségrégation, qui sont perçues comme nécessaires à l'intégration des hommes, des secteurs d'activité et des territoires de la ruralité.

Nous montrons ainsi que la situation calédonienne est particulièrement illustrative $d u$ caractère explicatif d'une dialectique I/S touchant tout à la fois à l'espace, à l'économique et au social, mais aussi qu'elle oblige à nuancer la dichotomie apparente entre les termes et permet par là même de répondre à nos hypothèses.

\section{Les politiques agricoles et rurales de la Nouvelle-Calédonie au prisme de l'intégration et de la ségrégation}

\section{Introduction au terrain : contexte historique}

Succédant à une colonisation de peuplement pénale, agricole et minière sous le contrôle de l'État (1853-1946) et au passage au statut de territoire d'outre-mer (19461965), les années 1970 et 1980 sont marquées par les revendications indépendantistes kanak, avec un durcissement du conflit jusqu'à une situation de quasi-guerre civile (période dite des «Événements » entre 1984 et 


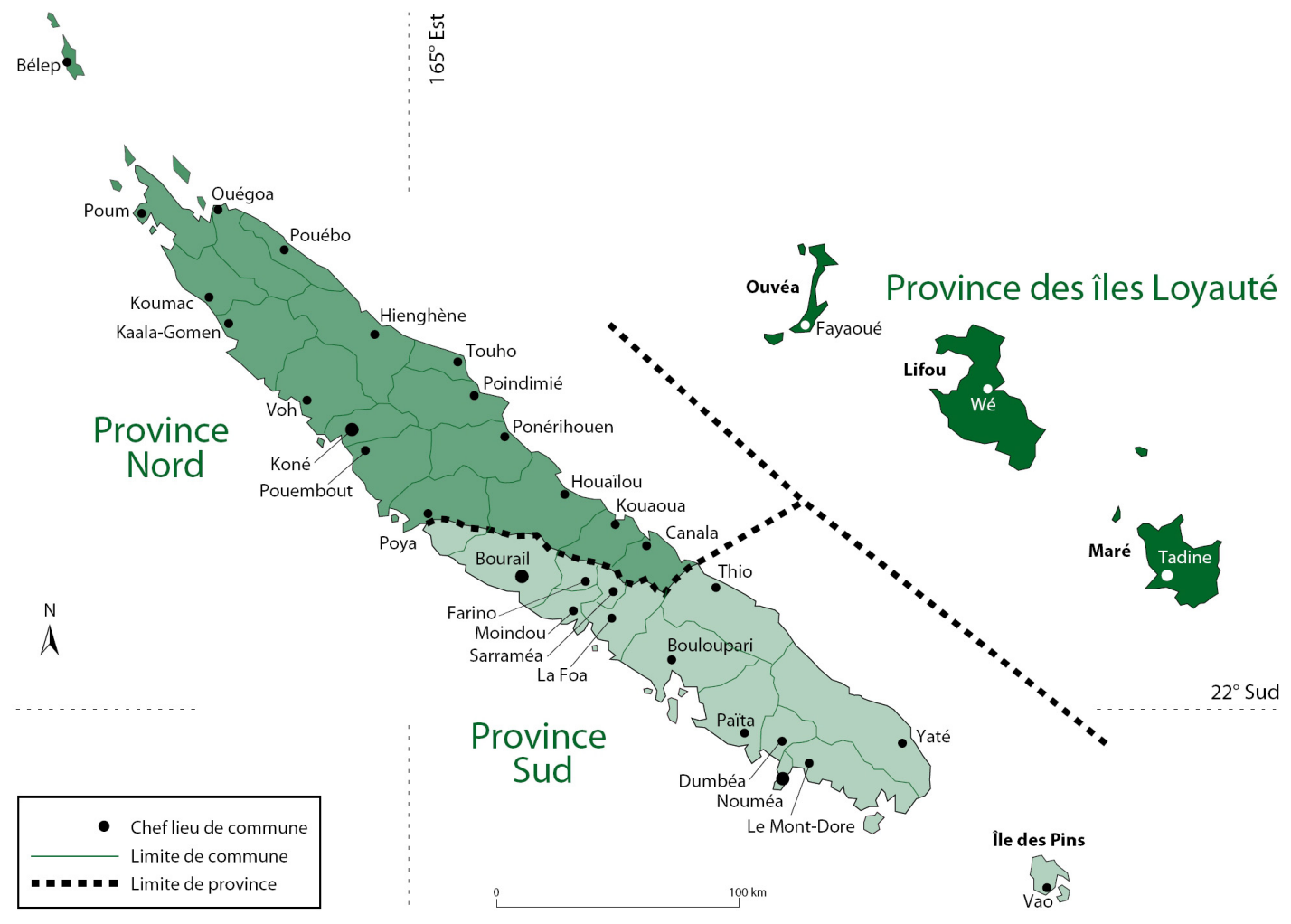

Fig. 1. Provinces et communes de Nouvelle-Calédonie (source : G. Pestaña).

1988). Voyant les impasses de la lutte armée, les deux camps (loyaliste et indépendantiste) se mettent d'accord pour une solution négociée, qui prend la forme d'une innovation institutionnelle instaurant un statut sui generis. Par les accords de Matignon (1988), le Premier ministre français de l'époque, Michel Rocard, entreprend de créer ce que l'on pourrait appeler un «État fédéral » avec un congrès propre à la Nouvelle-Calédonie qui coordonne les actions de trois provinces (Fig. 1). Par le jeu des découpages électoraux, deux d'entre elles principalement peuplées de Kanak (Nord et îles Loyauté) auront un exécutif élu indépendantiste, tandis que la troisième, incluant la ville de Nouméa (Sud), restera non indépendantiste.

Les compétences de l'État français sont limitées aux compétences régaliennes auxquelles sont ajoutés le droit du travail et l'enseignement du second degré et supérieur. Le territoire (la Nouvelle-Calédonie) a compétence pour l'élaboration de son budget, la fiscalité et les travaux d'intérêt territorial. L'État français s'engage à fournir des moyens importants aux nouvelles collectivités locales à travers l'élaboration de contrats de développement et propose que les produits financiers issus de l'exploitation du nickel viennent compléter ses transferts publics. En province Nord en particulier, les trois contrats de développement qui se sont succédé depuis 1990 ont eu pour objectif de favoriser les conditions du développement par un rattrapage en matière d'infrastructures.

Initialement prévu pour dix ans, le dispositif des accords de Matignon-Oudinot sera reconduit en 1998 pour quinze ans par l'accord de Nouméa. Ce dernier renforce encore les pouvoirs de chaque province et instaure un gouvernement collégial. Au terme du processus d'émancipation que consacre ce nouvel accord, le rôle de l'État français se cantonnera aux obligations monétaires et militaires. L'accord de Nouméa peut ainsi être perçu comme une tentative d'intégration économique et sociale en réponse à la ségrégation opérée par le passé et qui s'est notamment manifestée par la mise en réserve du peuple kanak et la contrainte du bagne pour les premiers colons qui ont participé, malgré eux, à cette colonisation de peuplement.

« Le moment est venu de reconnaître les ombres de la période coloniale, même si elle ne fut pas dépourvue de lumière. [...] Des clans ont été privés de leur nom en même temps que de leur terre. [...] les mouvements de population l'ont déstructurée [1'organisation sociale kanak], la méconnaissance ou des stratégies de pouvoir ont conduit trop souvent à nier les autorités légitimes. [...] À cette négation 
des éléments fondamentaux de l'identité kanak se sont ajoutées des limitations aux libertés publiques et une absence de droits politiques [...] » (accord de Nouméa, préambule).

Dans cette recherche d'une vision intégrationniste, deux principes sont remarquables :

1. Le principe de "rééquilibrage" vise un développement plus harmonieux des infrastructures sur l'ensemble du territoire et une répartition plus équitable des richesses, de l'accès aux services de base et surtout du pouvoir.

« Des contrats de développement sont conclus entre l'État et les provinces afin de financer conjointement des actions destinées à atteindre, en prenant en compte les spécificités provinciales, des objectifs de développement dont les principaux sont $[\ldots]: 1$. Faciliter l'accès de tous aux formations initiales et continues et adapter celles-ci aux particularités du territoire $[\ldots] ; 2$. Favoriser un rééquilibrage du territoire par rapport à l'agglomération chef-lieu et améliorer les infrastructures pour permettre le désenclavement des populations isolées $[\ldots] ; 3$. Améliorer les conditions de vie des populations de toutes les parties du territoire [...] ; 4. Promouvoir le patrimoine culturel mélanésien et celui des autres cultures locales » (accords de Matignon, 1988, Titre VIII).

Il apparaît ainsi nécessaire de ségréguer territorialement les politiques et les moyens pour conduire une politique d'intégration à plus long terme, processus que nous qualifierons de ségrégation positive ${ }^{2}$. En ce sens, différentes mesures emblématiques sont ainsi mises en place, parmi lesquelles :

- la mise en place d'une clé de répartition favorisant budgétairement les provinces indépendantistes (Nord et Iles) ${ }^{3}$;

- l'affirmation de la souveraineté territoriale de chaque province, qui met en œuvre des politiques de développement différenciées et adaptées ;

- le gel du corps électoral, qui n'autorise que les participants au vote de l'accord de Nouméa en 1998 à se prononcer, à partir de 2014, pour la souveraineté, évitant ainsi toute stratégie d'immigration à des fins électoralistes.

2. De la reconnaissance des torts occasionnés par la colonisation de peuplement - notamment envers le peuple kanak - émerge le principe de «destin commun» (accord de Nouméa, 1998). Il invite toute la population à faire unité et à construire ensemble l'avenir.

\footnotetext{
2 Nous considérons que la ségrégation positive est un découpage (des catégories sociales ou culturelles, de l'espace, du territoire) ayant pour finalité d'obtenir plus rapidement des objectifs intégrationnistes.

3 Le rééquilibrage des budgets est inscrit dans la loi référendaire du 8 novembre 1988. Celle-ci stipule la répartition des dotations $d$ 'investissement suivante: $3 / 4$ pour les provinces Nord et Îles, 1/4 pour la province Sud.
}

«Des signes identitaires du pays, nom, drapeau, hymne, devise, graphisme des billets de banque, devront être recherchés en commun, pour exprimer l'identité kanak et le futur partagé par tous » (accord de Nouméa, art. 1.5).

Ces deux principes ont notamment permis la reconnaissance et la légitimation de catégories de population jusqu'alors marginalisées. Tout en obligeant la coopération entre les partis politiques à travers la mise en place d'un gouvernement collégial, l'accord de Nouméa répond ainsi à la demande d'autonomie du peuple kanak en mettant en place des politiques publiques différenciées et en transférant de nombreuses compétences aux collectivités locales.

Pour autant, l'explicitation de la politique agricole calédonienne montre que cette vision intégrationniste, qui fait sens politiquement, n'échappe pas au poids de l'histoire et à la permanence des ségrégations. Les visions intégrationnistes et ségrégationnistes ne correspondent qu'à une représentation d'une réalité plus complexe, dans laquelle les limites et les frontières restent mobiles, perméables, et le produit d'une construction sociale. Dès lors, le cas calédonien illustre la volonté rendue nécessaire par les dépendances de sentier - de dépasser cette dualité intégration/ségrégation en proposant d'autres voies.

\section{Les politiques agricoles : vers une ségrégation positive pour une intégration à (long) terme ?}

Avant 1988, seuls les Calédoniens d'origine européenne se voyaient proposer une politique agricole répondant à leurs attentes et à leurs besoins. En effet, marquée par une économie assistée (Freyss, 1995) et une forte urbanisation entre 1965 et 1984, l'agriculture perd de son importance 4 et de son poids dans le PIB (passe de $10 \%$ en 1960 à $2 \%$ en 1984), et devient durablement un secteur peu stratégique au profit de l'exploitation minière du nickel qui connaît un essor considérable à cette même période. Dans cette situation, l'élevage bovin européen des grandes exploitations, qui occupe plus de $70 \%$ de la surface agricole utile (SAU), est ainsi soutenu politiquement avec une volonté de « contrôle public des filières et des prix à la fin de la période » (Sourisseau et al., 2010, p. 55), laissant aux Kanak une agriculture de production vivrière, au mieux « améliorée ${ }^{5}$.

Conjointement, dès les années 1970, l'urbanisation croissante permet le développement de structures de production maraîchère intensive par des agriculteurs européens en périphérie de Nouméa et les filières

\footnotetext{
4 Selon l'Institut de la statistique et des études économiques, la production marchande aurait chuté, en euros constants, de 33,5 millions à 12,6 millions entre 1965 et 1981 .

5 Profitant de la redistribution des terres, certains Kanak orientent leur production vers le marché et se regroupent.
} 


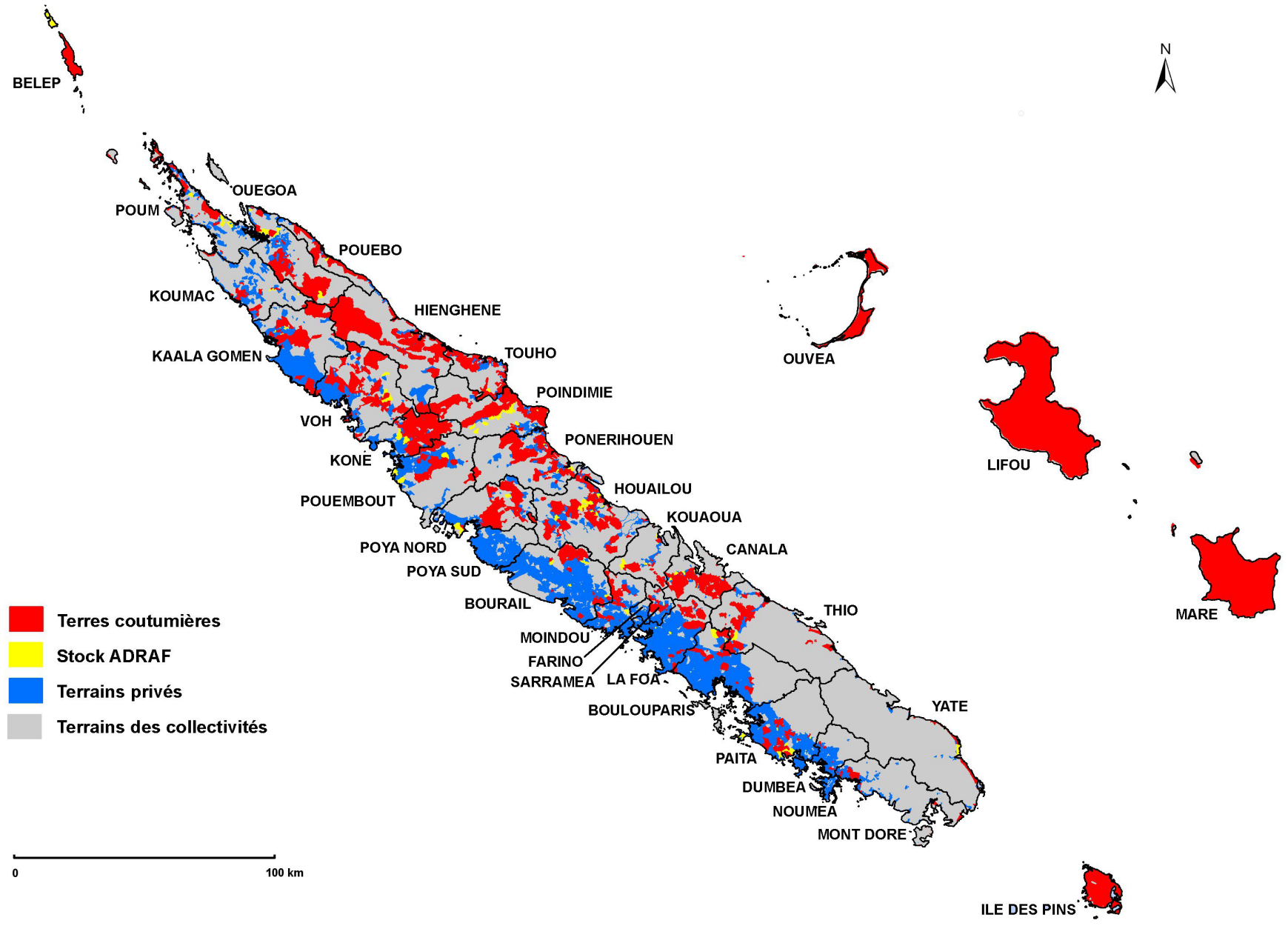

Fig. 2. Répartition du foncier en décembre 2008 (source : Adraf). Une figure en couleur est disponible à : www.nss-journal.org

«marchandes » bénéficient d'une protection via des régimes douaniers maîtrisés par les producteurs concernés. Entre 1970 et 1990, la dualité des politiques agricoles s'affirme, les pouvoirs publics essayant de répondre aux revendications de plus en plus fortes et audibles du peuple kanak, notamment en ce qui concerne la redistribution des terres à leur profit.

Dans un premier temps, seules des mesures économiques sont proposées, alors que les revendications sont principalement politiques. Prônée dès la fin des années 1970, l'intégration agricole (et plus largement économique) est alors synonyme d'insertion : les Kanak sont invités à reproduire les pratiques européennes et à s'inscrire dans l'arsenal français des aides à la modernisation agricole.

À partir de la fin des années 1980, ce processus d'intégration change $d$ 'aspect en laissant progressivement place au développement d'une nouvelle voie pour l'agriculture kanak avec des politiques de développement local qui redéfinissent les places et rôles de l'agriculture.
Les critères de productivité et de rentabilité économique laissent place aux notions d'autoconsommation, d'identité et de maintien des fonctions sociales de la production agricole kanak. Les acquis de la réforme foncière après les accords de Matignon-Oudinot illustrent ce changement d'orientation. Au nom du lien à la terre, 112000 ha ont été reversés dans le domaine des terres coutumières depuis 1988, conduisant à un rééquilibrage de la répartition entre terres privées et coutumières du foncier non affecté aux collectivités, tout en renforçant la partition de l'espace en foncier de statut très différent (Fig. 2).

Les types d'agricultures pratiquées sur ces deux fonciers, privé et coutumier, sont différents à bien des égards. Malgré le rééquilibrage en termes de surface, le recensement général de l'agriculture de $2002^{6}$ montre que ces territoires agricoles sont le siège de modèles distincts (Tab. 1 et Fig. 3). La SAU totale représentait $13 \%$

\footnotetext{
6 Les chiffres du dernier recensement général de l'agriculture réalisé en 2012 ne sont pas encore disponibles.
} 
Tableau 1. Agricultures duales sur terres coutumières et foncier privé.

\begin{tabular}{|c|c|c|}
\hline Terres privées & 2002 & Terres coutumières \\
\hline $29 \%$ & Part des agriculteurs & $71 \%$ \\
\hline $31 \%$ & Part des exploitations & $69 \%$ \\
\hline $74 \%$ & Part de la surface agricole utile & $26 \%$ \\
\hline 115 ha & Surface moyenne des exploitations & 14 ha \\
\hline
\end{tabular}

Source : Isee-Davar, 2005.

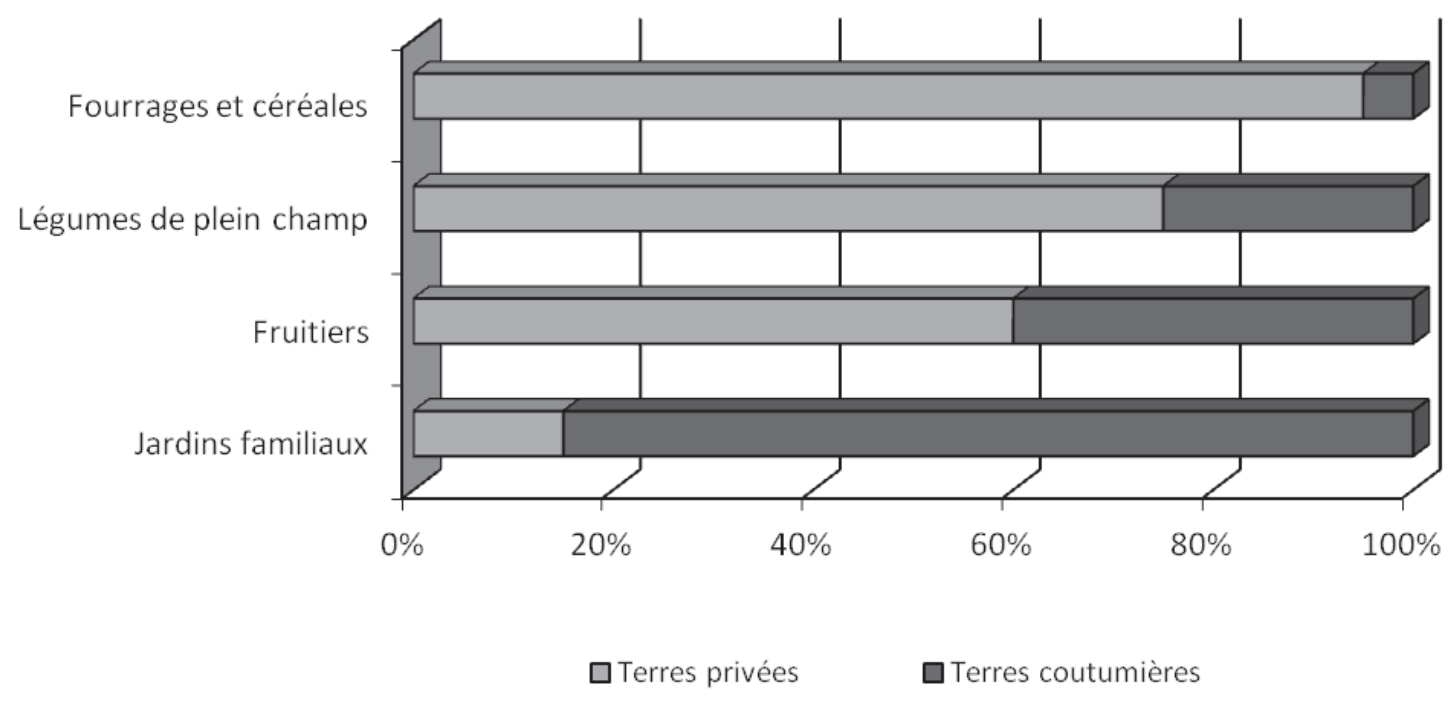

Fig. 3. Répartition des cultures entre terres privées et coutumières en 2002 (source : Isee, Davar, 2005).

de la surface totale de la Nouvelle-Calédonie, soit 248000 hectares, et $97 \%$ de cette SAU étaient des pâturages, supports de l'élevage extensif directement issu des logiques de la colonisation agricole, avec une forte concentration sur la côte ouest de la Grande Terre.

L'agriculture sur terres coutumières - et donc essentiellement kanak - concernait $69 \%$ des exploitations pour à peine un quart de la SAU, dont $85 \%$ étaient des jardins familiaux. Une étude plus récente sur l'agriculture sur terres coutumières montre que le recensement général de l'agriculture (RGA) sous-estime les productions kanak, notamment parce que celles-ci concernent de très petites surfaces par famille, que les itinéraires techniques sont peu consommateurs d'intrants et ont pour fonctions premières l'autoconsommation et le don, seuls $12 \%$ des produits agricoles étant effectivement vendus (Guyard et al., 2013).

Articulés à cette ségrégation spatiale, des dispositifs spécifiques d'appui au développement rural sont mis en œuvre. Les Ogaf (opérations groupées d'aménagement $\mathrm{du}$ foncier) sont ainsi le principal dispositif qui accompagne la réforme foncière. Les Ogaf concernent en effet principalement les terres coutumières, et notamment les groupements de droit particulier local (GDPL) ${ }^{7}$, qui constituent des zones intermédiaires où s'exercent le droit coutumier, mais où des possibilités de développement sont plus ouvertes. Ce dispositif permet de verser des aides à caractère incitatif (primes, contrats, subventions, etc.) pendant une durée de trois à cinq ans. À l'instar de nombre d'opérations mises en œuvre en Europe (cf. les programmes Leader), un suivi de l'opération est réalisé par des comités rassemblant les acteurs locaux (Bouard, 2013). Depuis 1993, huit Ogaf ont été engagées sur la partie est de la Nouvelle-Calédonie, où se concentrent la majorité des terres coutumières réattribuées dans le cadre de la réforme foncière (Fig. 4).

\footnotetext{
7 La plupart des GDPL fonciers ont été constitués à partir du début des années 1990 en vue de bénéficier des attributions foncières effectuées par l'Agence de développement rural et d'aménagement foncier d'État, sous le régime du droit coutumier.
} 


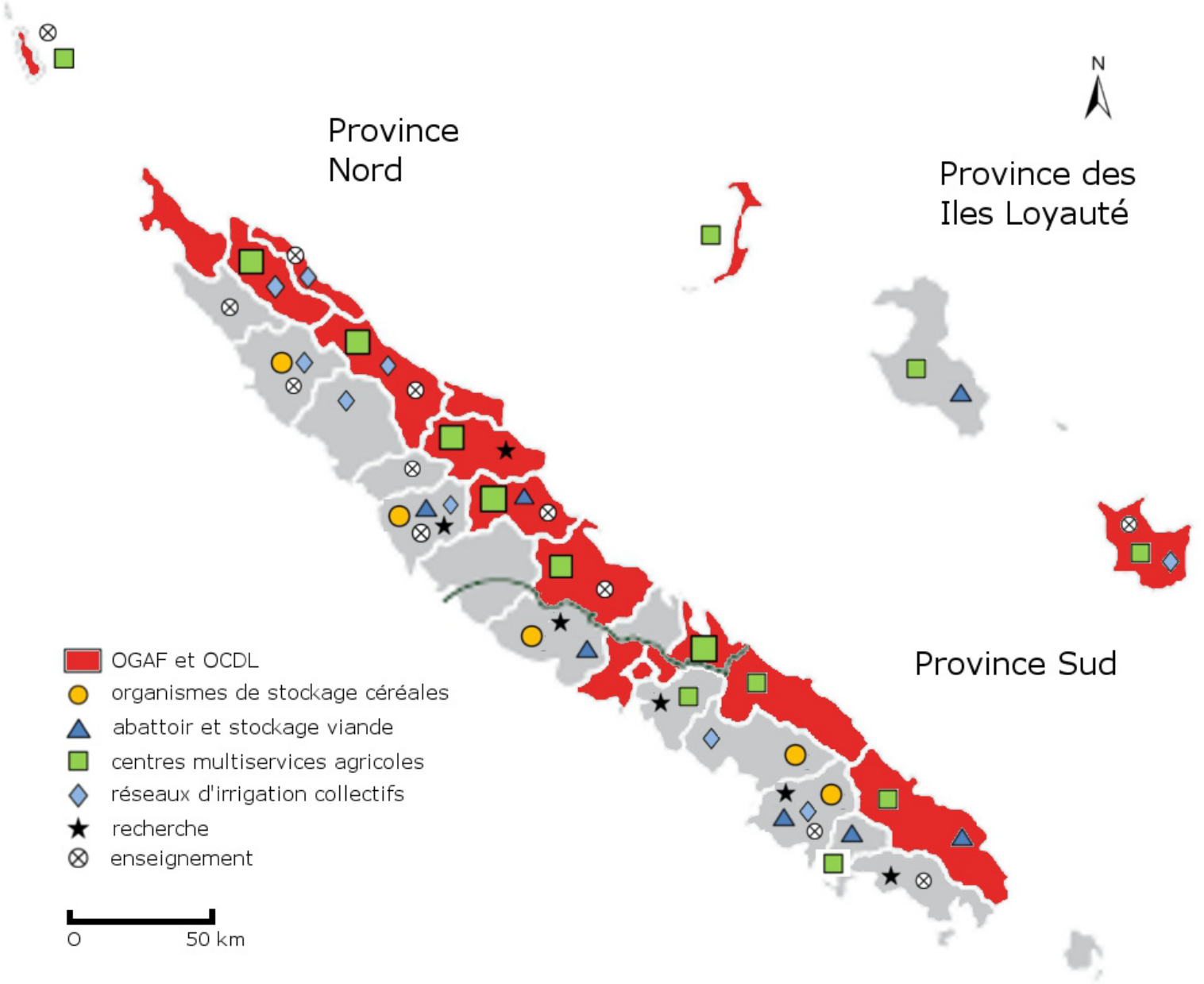

Fig. 4. Infrastructures agricoles, collectives ou publiques.

En province Nord, conjointement aux Ogaf, le code de développement permet des interventions sur terres coutumières et foncier privé. Trois catégories d'aides sont explicitement distinguées pour appuyer le développement : les aides à l'entreprise, les aides aux projets d'insertion (rééquilibrage) et les aides aux projets traditionnels. Cette distinction renforce la ségrégation entre projets économiques, en segmentant de manière forcée des fonctions et objectifs spécifiques assignés aux différentes activités économiques et en particulier à l'agriculture.

Ces évolutions renforcent le caractère dual des politiques agricoles. D'un côté des mesures visent à renforcer l'aide à l'investissement et à la protection des prix pour l'agriculture de type européen et, de l'autre, des opérations de développement local valorisent l'agriculture kanak, notamment sur terres coutumières. Cette dualité assumée n'exclut cependant pas le maintien d'inégalités et se caractérise par un faible budget consacré au développement local (10 \% à peine). Les rigidités et dépendances de sentier issues de la colonisation de peuplement rendent très difficile le dépassement de ces différenciations. Dès lors, un dualisme raisonné et non excluant apparaît comme une solution crédible.

\section{Les recompositions sociales dans le monde rural : dépasser la dualité intégration/ségrégation}

La clé de lecture I/S apparaît également utile pour analyser finement les recompositions sociales et spatiales et surtout, invite à se pencher sur les flux qui traversent les frontières et bousculent l'opposition entre intégration et ségrégation.

\section{La montée en puissance d'un capitalisme kanak d'entreprise}

Les dynamiques récentes internes au monde kanak témoignent de changements rapides et de l'hybridation 
permanente entre les représentations séparées du développement issues de la colonisation. Jusqu'à récemment, et malgré quelques expériences éparses issues d'actions militantes dans les années 1970, le monde de l'entreprise exclut de fait les Kanak des responsabilités. Les élites économiques, soucieuses de leurs prérogatives, entretiennent un modèle d'entreprise hérité d'une logique de comptoir, fonctionnant sur une connexion forte au politique et sur des prix élevés permis par les transferts publics, notamment à travers la forte présence d'une administration d'État et locale. Le patronat, dans un réflexe "ségrégationniste », considère qu'il revient aux Kanak d'intégrer ses principes d'entreprise, partant du principe que leurs pratiques sociales sont faiblement compatibles avec un capitalisme efficace. Mais depuis la provincialisation et l'ouverture des politiques économiques aux Kanak, ces derniers se sont progressivement mais très rapidement adaptés aux opportunités offertes par une conjoncture favorable. Ils ont modifié certains de leurs comportements tout en maintenant un lien étroit avec leurs traditions communautaires.

Deux exemples, issus du monde de la mine, mais impactant très directement les dynamiques rurales, illustrent les capacités d'innovation de ce capitalisme kanak naissant.

Le premier concerne une entreprise de remorquage développée et gérée par un clan dans le cadre du chantier métallurgique du Nord (Poithily, 2010, p. 27). Ses dirigeants, afin de rentabiliser leur activité, ont utilisé les codes et les techniques occidentales de management de l'entreprise. Pour autant, les dirigeants et les employés continuent d'entretenir une logique redistributrice en direction des familles de leur communauté, dès lors que la stabilité des relations partenariales avec « l'extérieur » est garantie. Deux types de relations, deux modes de gestion et de management se mettent en place, selon que l'entreprise traite avec ses clients externes dans la recherche et la conduite des contrats, ou avec ses alliés et sa famille pour la mobilisation de la force de travail. Une même dualité est observée pour l'utilisation et la redistribution des revenus de l'activité.

Le second exemple concerne la $\mathrm{SMSP}^{8}$ qui, pour charger le minerai sur l'un de ses sites, sous-traite cette activité à deux sociétés à actionnariat populaire issues de trois villages kanak proches du lieu de chargement (Sourisseau et al., 2006). Plutôt que de mettre en place des contrats individuels classiques, la SMSP traite avec des collectifs sur service rendu. Cet arrangement permet d'avoir une main-d'œuvre toujours disponible

\footnotetext{
8 Société minière du Sud Pacifique, entreprise minière dont la province Nord, à travers sa holding Sofinor, est l'actionnaire majoritaire. La SMSP est l'entreprise emblématique d'un capitalisme public kanak susceptible de porter un modèle économique viable dans un contexte d'indépendance.
}

pour répondre à ses besoins et aux membres des clans concernés de capter la rente du chargement tout en préservant les logiques collectives de répartition du travail. La régularité et l'assiduité difficiles à obtenir dans un cadre individuel sont dès lors gérées à l'échelle du groupe. Les clans s'organisent ainsi pour que l'activité de sous-traitance soit assurée, sans trop pénaliser les activités qu'ils réalisent traditionnellement dans leur communauté.

\section{L'évolution des fonctions de l'activité agricole et la participation aux programmes de développement local pour les ménages kanak}

Fondamentalement tournée vers l'autoconsommation et l'entretien des relations interpersonnelles entre les familles et les clans, l'agriculture kanak exprime de manière originale ses fonctions marchandes (Bouard et Sourisseau, 2010). Mais cette apparente immobilité face à la progression des activités salariées masque des dynamiques contradictoires, au cœur des tensions internes et externes à la société kanak entre aspiration à l'insertion (marchande et sociale) et repli communautariste. Les trajectoires et les stratégies de deux agriculteurs illustrent quelques facettes de ces formes d'hybridation.

Ces deux agriculteurs faisaient partie d'un GDPL et ont pu récupérer des droits fonciers leur permettant de développer une agriculture professionnelle et marchande. Conviés tous les deux à devenir membres d'un comité local de discussion ouvert par une Ogaf sur leur commune, ils n'ont pas fait le même choix.

\section{Cas A :}

Le ménage A pratique une agriculture «professionnelle » et marchande. Installé sur un GDPL, il est spécialisé dans la production d'ignames et de taros d'eau depuis 1994. La quasi-totalité de sa production est commercialisée et il possède une petite entreprise de fauchage et d'entretien des bas-côtés des routes.

En s'investissant dans une activité agricole marchande, le ménage s'est progressivement désengagé des activités sociales menées par son clan et sa tribu, ainsi que des cérémonies coutumières : «On fait la part des choses [...], il faut gérer entre les obligations sociales et le travail. »

Suite à la mise en œuvre d'une Ogaf, le ménage (à travers la femme) s'est impliqué dans un des comités locaux de discussion ouverts par le projet. Celui-ci a eu accès à l'information nécessaire au montage de microprojets financés par le programme et a fait valoir les intérêts des membres du GDPL. Il a diffusé cette information au sein de son clan pour favoriser des installations sur de nouvelles parcelles, puis a aidé, grâce à ses contacts avec les commerçants, les personnes se 
lançant dans la commercialisation des productions issues des parcelles appuyées par l'Ogaf.

Dans cet exemple, le ménage négocie la coexistence de différents types de projets agricoles à vocation plus ou moins marchande et professionnelle sur le même espace foncier, le GDPL du clan. En favorisant l'accès à des microprojets pour les autres membres de leur clan ou de leur tribu qui ne pratiquaient pas une agriculture professionnelle et marchande, le ménage légitime le maintien de ses propres pratiques agricoles et l'espace foncier qu'il utilise à ce titre.

\section{Cas B :}

L'exploitant agricole du ménage B a d'abord travaillé comme soudeur dans des entreprises de Nouméa. Après les «Événements » des années 1980, il décide de s'installer en tant qu'agriculteur sur les terres de son clan. Il commence par la multiplication de semences d'igname pour les échanges coutumiers puis bifurque vers la production d'ignames pour la commercialisation dans les années 1990. Mais sa famille l'oblige à quitter la réserve familiale, et il doit louer des parcelles sur du foncier privé à proximité de sa tribu. Depuis maintenant dix ans, il passe la totalité de son temps à travailler des terres de droit commun situées à l'entrée de la vallée où il est domicilié et les relations avec son clan se sont distendues. Comme dans le cas du ménage A, l'exploitant est devenu membre d'un des comités locaux ouvert par l'Ogaf. Cependant, dès la troisième réunion, il quitte le comité car «en désaccord» avec les objectifs et la démarche du projet.

Spatialement et socialement marginalisé par son clan à cause de son activité agricole marchande, il n'a pas pu participer au comité local de manière effective. Dès lors le choix est fait d'une séparation de fait entre agriculture et vie sociale. Pourtant souvent cité comme un exemple de réussite de l'agriculture kanak, l'exploitant B est seul à promouvoir le modèle de l'agriculture spécialisée, professionnelle et marchande.

Ces exemples montrent comment les acteurs tendent à articuler en les maintenant plus ou moins fortement séparées, les pratiques sociales propres au monde kanak et les exigences d'une activité économique régie par des conventions relevant plutôt du monde de l'entreprise et donc plus empreinte de normes occidentales. Cette double posture vise à élargir le champ des possibles en se saisissant des incitations d'un marché dopé par une forte croissance, tout en ne se coupant pas des jeux de pouvoir internes à la tribu et aux clans, voire en se laissant la possibilité de les valoriser dans le monde de l'entreprise. Ces recompositions ou leur absence sont le reflet de frontières qui sont économiquement et socialement de plus en plus mobiles et poreuses.

\section{Intégration/ségrégation : vers une lecture renouvelée des politiques de développement?}

Si la clé de lecture I/S est intéressante pour comprendre le rôle de l'action publique sur les transformations de la ruralité calédonienne, la réalité dépasse les représentations intégrationnistes et ségrégationnistes que nous lui prêtons. Les études de cas proposées valident nos trois hypothèses, invitent à dépasser le modèle dichotomique et à redéfinir de façon élargie les notions d'intégration et de ségrégation.

Avant de revenir sur nos hypothèses, il convient de souligner que la Nouvelle-Calédonie illustre, sans doute à l'extrême, d'autres situations fréquentes de ségrégation « négative » dans l'espace colonial ou dans des situations de faiblesse extrême du fonctionnement démocratique. De telles formes instituées de ségrégation, pensées comme partie intégrante d'un modèle de développement, ont des coûts humains et sociaux, qui finissent généralement par les rendre inopérantes et obligent à les faire évoluer.

La Nouvelle-Calédonie post-«Événements » porte l'image d'une intégration, médiatisée dans une version « positive » elle aussi archétypique, venant en réparation des ségrégations passées. Le destin commun en particulier fixe un cap idéologique d'une intégration des valeurs et des identités, qui n'est pas sans rappeler, pour n'en citer que deux, les évolutions récentes constatées en Australie et en Afrique du Sud.

Mais en Nouvelle-Calédonie comme ailleurs, la réalité est autre que l'affichage humaniste proposé ; l'intégration prend des formes plus complexes que la communauté de destin. Les ségrégations issues des rapports de force coloniaux perdurent, mais elles acquièrent aussi une dimension compensatrice et in fine intégratrice. Il y a bien ségrégation dans la mesure où des espaces, des acteurs, des politiques sont volontairement différenciés et se définissent dans leur opposition, mais aussi lorsque des catégories d'acteurs se voient effectivement dédier des politiques publiques et des espaces spécifiques. Mais il y a également intégration du fait que des catégories de la population, jusqu'ici exclues des dispositifs de développement, deviennent les cibles des politiques publiques, et que les sociétés ménagent des passerelles entre les espaces et les acteurs économiques, rompant ainsi non pas avec la dualité qui est revendiquée, mais avec l'étanchéité entre les catégories. La spécialisation affichée de ces espaces et la liberté donnée à l'invention et à l'adaptation des instruments multiplient les combinaisons possibles, et les représentations du développement et de ses normes. 
Ainsi est démontré l'intérêt de concevoir I/S comme les faces d'un même prisme ; limiter l'interprétation de l'action publique provinciale à la mise en place de dispositifs favorisant le processus d'intégration des populations kanak dans l'agriculture marchande n'est pas suffisant.

Par ailleurs, quelle que soit l'échelle d'observation privilégiée, la mise en perspective historique des politiques foncières et de développement en Nouvelle-Calédonie depuis la colonisation témoigne d'alternances entre des phases d'intégration et de " ségrégation compensatrice ». La diversité révélée des trajectoires et pratiques des individus, qui sont pourtant confrontés à la promotion d'une bifurcation politique intégratrice, valide notre deuxième hypothèse : la diffusion d'un modèle agricole n'est pas un processus linéaire.

En outre, les trajectoires de développement résultent d'actions et de réactions, soumises aux forces du marché et aux forces sociales et identitaires. Ces forces peuvent conduire à des trajectoires différenciées, qui invitent à relativiser les impacts anticipés de la mise en place d'instruments de politiques publiques.

Aussi, avec toutes les limites de ce type de comparaison, la ségrégation des publics et des territoires est finalement très fréquente dans les contextes postcoloniaux de peuplement (en Afrique du Sud, en Australie et sous d'autres formes au Brésil), dans le but d'intégrer aux choix globaux des espaces et des ressources jusqu'alors marginalisés et oubliés. Dans toutes ces situations, conformément à notre troisième hypothèse, c'est la question des frontières qui se pose dans l'accompagnement volontariste du développement. Les enjeux se focalisent sur la façon de définir quel acteur, quelle ressource et quel espace relèvent de tel ou tel dispositifs de politique publique (intégration) ou non (ségrégation).

Le cas calédonien insiste sur la difficulté de ces définitions, liée à la porosité et à la plasticité des frontières (Caron, 2011). Ces dernières ne sont que des représentations temporaires susceptibles d'évoluer avec les dynamiques sociales à l'œuvre. Elles ne sont pas que des lignes matérialisant une séparation; elles peuvent être des zones stratégiques où les innovations prennent forme. Ainsi, en Nouvelle-Calédonie, les GDPL sont des espaces d'intersection entre des représentations coutumières de repli et des ouvertures vers des logiques d'anonymisation que rend nécessaire l'insertion marchande. Ces exemples gagneraient à être généralisés ; la clé de lecture I/S peut aider à mettre en lumière une troisième frontière agraire appréhendée par Hubert et Billaud (2008).

Pour finir, la mobilisation du couple I/S relativise la réalité des grands modèles de développement agricole, et invite à une meilleure compréhension des idéologies et des pratiques. À défaut de fournir les moyens d'améliorer les politiques publiques de développement, cette clé permet de pointer la complexité des processus à l'œuvre et la possibilité de cohabitation vertueuse d'orientations souvent présentées comme antinomiques. Parmi les prolongements possibles à notre réflexion, une comparaison entre la Nouvelle-Calédonie, l'Afrique du Sud, l'Australie et sous certains aspects le Brésil permettrait de creuser les différentes voies et influences coloniales de la mise en politique d'une action publique compensatrice en milieu rural. Dans ce sens, le couple I/S peut aussi éclairer les recherches en géographie critique menées sur les processus de développement mais aussi de « racialisation » du développement (Glassman, 2010).

\section{Remerciements}

Les auteurs tiennent à remercier Patrick Caron pour la relecture attentive des versions préparatoires à cet article.

\section{Références}

Bouard, S., 2013. La ruralité kanak à la recherche d'un modèle décolonisé, Papeete, Tahiti, Au vent des Îles.

Bouard, S., Sourisseau, J.-M., 2010. Stratégies des ménages kanak: hybridations entre logiques marchandes et non marchandes, Natures Sciences Sociétés, 18, 3, 266-275 (online: http:/ /dx.doi.org/10.1051/nss/2010033).

Bullard, N., 2011. It's too late for sustainability: What we need is system change, Development, 54, 2, 141-142.

Caron, P., 2011. Ré-concilier agricultures et sociétés: dévoiler le territoire et repenser les limites. Mémoire de recherche pour l'habilitation à diriger des recherches, Université Paris Ouest-Nanterre La Défense, Paris.

Caron, P., Le Cotty, T., 2006. A review of the different concepts of multifunctionality and their evolution, European Series on Multifunctionality, 10.

Caron, P., Reig, E., Roep, D., Hediger, W., Le Cotty, T., Barthélémy, D., Hadynska, A., Hadynski, J., Oostindie, H., Sabourin, É., 2008. Multifunctionality: Epistemic diversity and concept oriented research clusters, International Journal of Agricultural Resources, Governance and Ecology, 7, 4-5, 319338, doi:10.1504/IJARGE.2008.020080.

Cary, P., Fol, S., 2012. Introduction : les métropoles face aux dynamiques de ségrégation et de fragmentation, Géographie, Économie, Société, 14, 2, 113-126.

Elias, N., Scotson, J.-L., 1997. Logiques d'exclusion, Paris, Fayard. Freyss, J., 1995. Économie assistée et changement social en Nouvelle-Calédonie, Paris, Presses universitaires de France.

Gautier, D., Ankogui-Mpoko, G.F., Réounodji, F., Njoya, A., Seignobos, C., 2005. Agriculteurs et éleveurs des savanes d'Afrique centrale: de la coexistence à l'intégration territoriale, L'Espace Géographique, 34, 3, 223-236 (online: http:/ / www.cairn.info/revue-espace-geographique-20053-page-223.htm). 
Gibson-Graham, J.K., 2010. Forging development partnerships: Possibilities for local and regional development, in Pike, A., Rodriguez-Pose, A., Tomaney, J., (Eds), Handbook of Local and Regional Development, London, Routledge, 226236.

Glassman, J., 2010. Critical geography II: Articulating race and radical politics, Progress in Human Geography, 34, 4, 506512, doi:10.1177/0309132509351766.

Graner, E., 2008. Integration versus fragmentation: Nepalese labourers in the global economy, Die Erde, 139, 3, 207-226.

Griffon, M., 2006. Nourrir la planète: pour une révolution doublement verte, Paris, Odile Jacob.

Guyard, S., Apithy, A., Bouard, S., Sourisseau, J.-M., 2013. Revenus des familles résidant en tribu en NouvelleCalédonie : la ressource non monétaire en question, Revue Juridique, Politique, Économique et Sociale de NouvelleCalédonie, 21, 100-110.

Hazell, P., Poulton, C. Wiggins, S., Dorward, A., 2007. The future of small farms for poverty reduction and growth, Discussion Paper 42, International Food Policy Research Institute (IFPRI) 2020, May 2007, Washington DC.

Hobbs, N.T., Galvin, K.A., Stokes, C.J., Lackett, J.M., Ash, A.J., Boone, R.B., Reid, R.S., Thornton, P.K., 2008. Fragmentation of rangelands: Implications for humans, animals, and landscapes, Global Environmental Change, 18, 4, 776-785.

Hubert, B., 2010. L'agronomie, science de l'agriculture ?, Le Mouvement Social, 233, 4, 143-157, doi:10.3917/lms.233.0143.

Hubert, B., Billaud, J.-P., 2008. Pour ne plus voir d'émeutes de la faim: une troisième frontière agraire à explorer?, Natures Sciences Sociétés, 16, 2, 109-110 (online: http:// dx.doi.org/10.1051/nss:2008032).

Hubert, B., Caron, P., 2009. Imaginer l'avenir pour agir aujourd'hui, en alliant prospective et recherche: l'exemple de la prospective Agrimonde, Natures Sciences Sociétés, 17, 4, 417-423 (online: http://dx.doi.org/ $10.1051 / \mathrm{nss} / 2009060)$.

IAASTD, 2009. Agriculture at a Crossroads: Synthesis Rapport, International Assessment of Agricultural Knowledge, Science and Technology for Development, London, IslandPress.

Isee-Davar, 2005. RGA terres coutumières. Rapport Isee-Davar, Nouméa, 84 p.

Koop, K., Landel, P.-A., Pecqueur, B., 2010. Pourquoi croire au modèle du développement territorial au Maghreb ? : une approche critique, EchoGéo, 13 (online: http://echogeo. revues.org/12065).

Léonard, É., 2005. Frontière interne, gouvernance locale et production de la culture politique en milieu rural mexicain : la réforme agraire dans le Sud-Veracruz, 19202000, Cahiers des Amériques Latines, 45, 51-74.

Losch, B., Fréguin-Gresh, S., White, E., 2011. Rural Transformations and Late Developing Countries in a Globalizing World. World Bank report, Washington DC.

Menzel, U., 1992. Das Ende der Dritten Welt und das Scheitern der Großen Theorie, Frankfurt am Main, Suhrkamp.
Merle, I., 1995. Expériences coloniales: la Nouvelle-Calédonie (1853-1920), Paris, Belin.

Morin, E., 1966. Adolescents en transition : classe adolescente et classes sociales, aspiration au divertissement et aspiration à la vie bourgeoise dans une commune du SudFinistère, Revue Française de Sociologie, 7, 4, 435-455.

Moustier, P., Anh, T.D., 2010. Promouvoir l'information et la coopération : l'accès des petits producteurs aux filières de qualité au Vietnam, Perspective (Policy Brief), Cirad, 5, 6.

Poithily, D., 2010. Les chefs d'entreprise de la zone Vook [Voh]Koohnê [Koné]-Pwembu [Pouembout] : stratégies et motivations dans le projet Koniambo. Mémoire de master "Aménagement et développement territorial ", Université de la Nouvelle-Calédonie, Nouméa.

Saussol, A., 1979. L'héritage : essai sur le problème foncier mélanésien en Nouvelle-Calédonie, Paris, Société des océanistes, Musée de l'Homme.

Scholz, F., 2005. The theory of fragmenting development, Geographische Rundschau International Edition, 1, 2, 4-11.

Sencébé, Y., 2009. Une lecture des inégalités et des différenciations de développement local à travers quatre figures de territoires, Espace Populations Sociétés, 1 (online: http:// eps.revues.org/3550).

Sourisseau, J.-M., Tyuienon, R., Gambey, J.-C., Djama, M., Mercoiret, M.-R. 2006. Les sociétés locales face aux défis du développement économique: province Nord de NouvelleCalédonie, Pouembout, IAC/DDEE-PN/Cirad.

Sourisseau, J.-M., Pestaña, G., Gaillard, C., Bouard, S., Mennesson, T., 2010. À la recherche des politiques rurales en Nouvelle-Calédonie: trajectoires des institutions et représentations locales des enjeux de développement (18532004), Nouméa, IAC Éditions, Études et Synthèses, Tabù Éditions.

Timmer, P.C., 2009. A World without Agriculture: The Structural Transformation in Historical Perspective, Washington DC., AEI press.

United Nations, 2000. Millenium Development Goals, New-York, United Nations.

Van der Ploeg, J.D., 2000. Revitalizing agriculture: Farming economically as starting ground for rural development, Sociologia Ruralis, 40, 4, 497-511, doi:10.1111/1467-9523. 00163.

Vieillard-Baron, H., De Almeida Vasconcelos, P., 2004. Une lecture de la ségrégation au Brésil au regard de la situation française, Diversité, 139, 171-178.

World Bank, 2007. World Development Report 2008: Agriculture for Development. Washington DC, World Bank.

Zenou, B., 2011. La dualité intégration/ségrégation est-elle une clé de lecture utile pour discuter des transformations et des recompositions aux Suds? Rapport pour le département Environnements et sociétés, Cirad, Montpellier.

Zidouri, F., 2008. Gouvernance sociale et inclusion sociale à partir du projet d'autonomie régionale du Sahara, in $\mathrm{Le}$ projet marocain d'autonomie de la région du Sahara: garantie et viabilité, Centre d'études internationales, 202-208. 